cognitive tests: verbal memory (recall of 10 words), verbal fluency (naming animals) and letter search speed. Fully adjusted models included health and lifestyle covariates (medical history, depression, alcohol, physical activity).

Results Broadly similar patterns of associations were observed across countries. Indicators of SEP across the lifecourse made independent contributions to cognition, with stronger contributions from education and current asset ownership (an indicator of contemporary material circumstances), than from childhood socioeconomic conditions. Socioeconomic advantage was associated with higher levels of cognitive functioning. For example, in fully adjusted models, university education was associated with 3.9 (SE 0.7) word recall advantage in memory performance in Czech men and 2.2 (SE 0.5 ) and 2.7 (SE 0.3) word advantage in Russian and Polish men; in women these figures were 3.7 (SE 0.4), 3.0 (SE 0.5) and 3.2 (SE 0.3), respectively. The effect of childhood socioeconomic conditions on cognition was largely attenuated after adjustment for education and current material circumstances, suggesting mediation by later SEP measures.

Conclusion Socioeconomic position across the lifecourse is an important predictor of cognition in mid and later life in these Eastern European populations. The attenuation of the effects of childhood SEP after adjustment for own education and current material circumstances supports the model where this association is, at least partly, mediated through later life measures of SEP. Future research should focus on lifecourse influences on cognitive aging trajectories as long-term follow-up of this cohort and other studies in Eastern European populations become available.

\section{OP43 ARE WE OVERESTIMATING THE BENEFICIAL EFFECTS OF ALCOHOL IN LATER LIFE? THE CASE OF YOUNG NON- DRINKERS}

doi:10.1136/jech-2012-201753.043

L Ng Fat. Department of Epidemiology and Public Health, UCL, London, UK

Background Non-drinkers have been repeatedly shown to have worse health than moderate drinkers in later life particularly as regards conditions such as coronary heart disease, leading some researchers to suggest that moderate alcohol consumption has beneficial effects on health. However the causal direction between nondrinking and worse health has been contested. Whether poor health is associated with non-drinking among young adults in relation to social and health factors will be investigated. Such a finding would imply that poor health may precede non-drinking even at early stages of the life course.

Methods We performed logistic regression analysis of cross-sectional national survey data on 18 to 34 year olds, collected from The Health Survey for England 2006 and 2008. Data was collected through face to face interviews and is self-reported. Logistic regression analysis of longitudinal data collected from the National Child Development Study 1958 were also conducted. This is a follow up study to assess whether poor health at age 16 is associated with non-drinking at age 23 , and whether these people are more likely to abstain at subsequent age sweeps.

Results HSE 06 \& 08: Having a limiting longstanding illness during early adulthood increased the odds of being a non-drinker 1.74 times for men $(p<0.01)$, and 1.45 times for women $(p<0.01)$. In both men and women belonging to the lowest income quintile or having no qualifications was associated with increased odds of being a nondrinker $(p<0.001)$ indicating that the social gradient in non-drinking begins at an early age. Men and women aged 18 to 34 years with the lowest activity levels were also more likely to be non-drinkers $(p<0.01)$.

NCDS: Preliminary analysis reveals that non-drinkers at age 23 have higher rates of medical conditions at age 16 as assessed by a medical officer such as having a mental condition $(p<0.001)$ a physical condition and heart and haematological condition $(p<0.01)$.
Conclusion Young adults who have a limiting longstanding illness are more likely not to drink alcohol even after adjusting for a range of social and demographic measures. Studies on the putative health benefits of moderate alcohol consumption later in life need to take account of early life history. Further analysis using longitudinal data will explore whether poor health precedes non-drinking right at the start of drinking history and whether people with poor health continue to abstain from alcohol at older ages.

\section{OP44 RACE, BULLYING AND SELF-ESTEEM AT THE TRANSITION BETWEEN PRIMARY AND SECONDARY SCHOOL}

doi:10.1136/jech-2012-201753.044

A Hawkins, A Emond. Centre for Child and Adolescent Health, University of Bristol, Bristol, UK

Background Studies from the US have suggested that children who experience racial discrimination have higher rates of depression, anxiety, behavioural disorders, and lower self-esteem. Children are generally more vulnerable to such problems at the transition from one school to another. Our aim was to investigate the associations between race, bullying, mood, behavioural difficulties and selfesteem during the transition to secondary school in a population-based cohort of English children.

Methods Children from the Avon Longitudinal Study of Parents and Children (ALSPAC) were asked to define themselves by skin colour when aged 12 years $(n=7017)$. Analyses were performed using logistic regression, adjusting for gender and maternal education. The primary outcome measures were bullying and racial discrimination at $12-12.5$ years. Secondary outcomes were bullying at 8 years, depressive symptoms at 11.6 years, behavioural difficulties at 11.6 years, friendships at 12 years, mood and self-esteem at 13.8 years.

Results $94.2 \%$ of children defined themselves as white ( $\mathrm{n}=6607$ ), $3.6 \%$ as mixed race $(n=255)$ and $2.2 \%(n=155)$ identified with a specific ethnic minority group. At primary school (8-11 yrs), there were no differences between these groups in reported bullying, types of friends or prevalence of behavioural problems or depression. At secondary school (12.5 years), the reported prevalence of racially motivated violence was $10-13 \%$, and for name-calling was $31-33 \%$. Compared to white children, ethnic minority but not mixed race children were more likely to experience overt bullying (OR 2.98; $95 \%$ confidence interval 1.38 to 6.42 ). Mixed race children were more likely to retain friends of different races after the transition to secondary school (OR 1.89; 1.32 to 2.71 ). Ethnic minority children were more likely to feel 'different from others' at 13.8 years (OR $1.63 ; 1.01$ to 2.36 ).

Conclusion Although children of different colour had similar experiences at primary school, ethnic minority children were more likely than white children to experience bullying and discrimination at the transition to secondary school. Racial discrimination affected up to one third of ethnic minority and mixed race children at 12 years of age, and these children felt more socially isolated and were less happy than their white peers at 13 years. Strategies for prevention of bullying should be targeted at this vulnerable group of children, particularly during this high-risk period of transition.

\section{HSR: Quality And Outcomes of Care}

\section{OP45 CAESAREAN SECTION AND SUBSEQUENT FETAL DEATH: SYSTEMATIC REVIEW AND META-ANALYSIS}

doi:10.1136/jech-2012-201753.045

${ }^{1}$ SM 0 Neill, ${ }^{2}$ PM Kearney, ${ }^{3}$ LC Kenny, ${ }^{3}$ AS Khashan, ${ }^{4}$ TB Henriksen, ${ }^{1} J E$ Lutomski, 'RA Greene. 'National Perinatal Epidemiology Centre, University College Cork, Cork, Ireland; '2Department of Epidemiology and Public Health, University College Cork, Cork, 
Ireland; ${ }^{3}$ Anu Research Centre, University College Cork, Cork, Ireland; ${ }^{4}$ Perinatal Epidemiology Research Unit, Aarhus University Hospital, Aarhus, Denmark

Background Spontaneous miscarriage (the death of a fetus before 20 weeks of pregnancy), occurs in $10-15 \%$ of recognised pregnancies. Stillbirth (the death of a fetus after 24 weeks of pregnancy), occurs in approximately one in every 200 deliveries. The cause of miscarriage and stillbirth is frequently unknown. However, there is some evidence to suggest that previous Caesarean delivery may be a risk factor.

Objective: to compare the risk of fetal death in subsequent pregnancy by mode of delivery.

Methods This was a systematic review of relevant studies identified through CINAHL, the Cochrane library, Embase, Medline, PubMed, SCOPUS and Web of Knowledge (1945 - November 2011), using a comprehensive search strategy, and cross-checking of reference lists. Study selection: cohort and case-control studies reporting on Caesarean delivery and spontaneous miscarriage or stillbirth. Two reviewers independently assessed titles, abstracts, and full articles to identify eligible studies, using a standardised data collection form.

Results Miscarriage: From 6,857 titles identified, eight articles were included, totalling 147,017 women and 12,682 events. Odds ratios (ORs) were combined using a fixed-effect model to estimate the overall association using Review Manager Software. From the metaanalysis, the pooled OR estimate of miscarriage among women who previously delivered by Caesarean versus vaginally, was $1.11[95 \%$ CI 1.06,1.17]. The OR of miscarriage was 1.26 [95\% CI 0.54,2.92] for one case-control study, 1.11 [95\% CI 1.06,1.17] for seven cohort studies and 1.11 [95\% CI 1.06,1.17] for primiparous women (eight studies).

Stillbirth: From 6,857 studies identified, seven articles were included, totalling 1,661,335 pregnancies and 5,741 events. ORs were combined using a random effect model (due to the heterogeneity of included studies) to estimate the overall association. From the meta-analysis, the pooled OR estimate of stillbirth among women who previously delivered by Caesarean versus vaginally, was 1.32 [95\% CI 1.11,1.57]. The OR of stillbirth was 1.30 [95\% CI 1.03,1.64] for primiparous women (five studies), 1.40 [95\% CI 1.24,1.59] for multiparous women (two studies), 1.80 [95\% CI 1.27,2.55] for studies including all stillbirths (five studies) and, 1.20 [95\% CI 1.02,1.42] for studies including only unexplained antepartum stillbirths (three studies).

Conclusion Caesarean delivery compared to vaginal delivery is associated with an increased risk of spontaneous miscarriage by $11 \%$ and stillbirth by $32 \%$ in subsequent pregnancies. In light of the recently published National Institute for Health and Clinical Excellence (NICE) guidelines, which support a woman's right to request a Caesarean delivery without medical reason, there is an urgent need to establish whether mode of delivery has a causal effect on risk of fetal death.

\section{OP46 THE ASSOCIATION BETWEEN PRIVATE PATIENT STATUS AND CAESAREAN DELIVERY: A RETROSPECTIVE COHORT STUDY OF 403,642 CHILDBIRTH HOSPITALISATIONS}

doi:10.1136/jech-2012-201753.046

'JE Lutomski, ${ }^{2} \mathrm{M}$ Murphy, ${ }^{3} \mathrm{D}$ Devane, 'S Meaney, 1,2RA Greene. 'National Perinatal Epidemiology Centre, Cork, Ireland; 'Department of Obstetrics and Gynaecology, Cork University Maternity Hospital, Cork, Ireland; ${ }^{3}$ School of Nursing and Midwifery, National University of Ireland, Galway, Ireland

Background The increase in Caesarean delivery rates over the past decades has resulted in it becoming one of the most commonly performed in-hospital surgical procedures. While many Caesarean deliveries are clinically indicated, other factors, such as medico-legal fears and maternal and health professional preferences, contribute to the decision-making process. To explore non-clinical factors, we investigated differences in Caesarean delivery rates and temporal trends by private and public patient status while giving consideration to patient case-mix.

Methods A retrospective cohort study was performed on childbirth hospitalisations occurring between 2005 and 2010 in the Republic of Ireland. Procedural ICD-10-AM codes from hospital discharge records were used to identify emergency and elective Caesarean deliveries. Temporal trends in Caesarean delivery were determined using a Cochrane-Armitage test for trend. Multivariate multinomial regression analysis was used to determine the odds of Caesarean delivery (emergency or elective versus vaginal delivery) by mother's public or private status while adjusting for age, multiple gestation, previous Caesarean delivery, induction of labour, maternal morbidity and other obstetric complications.

Results 403,642 childbirth hospitalisations reviewed; approximately one-third of women (30.2\%) were booked privately. Women booked privately were more likely to be at least 30 years of age or older, married and have had a previous Caesarean delivery. Over the study period, the overall Caesarean rate increased from $22.2 \%$ to $23.8 \%$ among women booked publicly versus $30.2 \%$ to $34.7 \%$ among women booked privately (test for trend p-value $<0.0001$ ). While the emergency Caesarean rate was similar between both groups of women (14.3\% versus $13.3 \%$ respectively), women booked privately were almost twice as likely to have an elective Caesarean delivery (17.8\% versus $9.4 \%$ ). After adjustment, women booked privately had an increased odds of both emergency (adjusted OR: 1.32; 95\% CI $1.29,1.35$ ) and elective (adjusted OR: 1.88; 95\% CI 1.83, 1.93) Caesarean delivery.

Conclusion Irrespective of obstetric risk factors, women who opted for private maternity care were significantly more likely to have a Caesarean delivery than women booked publicly. Moreover, while increasing trends in Caesarean delivery were observed among all women, the increase was disproportionately higher among women booked privately. These findings suggest that significant differences in Caesarean rates are unlikely to be explained by differences in clinical risk factors. Mixed-method research is clearly warranted to explore disparities in Caesarean delivery rates. Such research should focus on clinical decision making and the role of personal preferences of women and maternity care professionals in decisions regarding mode of birth.

\section{OP47 EFFECT OF HIGH PROFILE QUALITY FAILURES ON PATIENT NUMBERS IN THE ENGLISH NHS}

doi:10.1136/jech-2012-201753.047

AA Laverty, C Millett. Department of Primary Care and Public Health, Imperial College London, London, UK

Background Policy architects are keen to emphasise that releasing information on the quality of hospitals into the public domain will drive patients away from poor quality providers and thus motivate system wide improvements. However, previous evidence on the impact of releasing quality information to the public have been mixed, with various reasons being proposed as possible barriers, including patients being unaware of the information available, being unable to understand it, or feeling it to be irrelevant. We examined three cases of high profile reports into failings in quality and safety at English NHS hospitals in order to assess whether this negative publicity was associated with a decrease in patient numbers. These reports all garnered significant media attention and presented a stark picture of performance and so we hypothesise that such negative coverage may have induced a fall in patient numbers.

Methods We analysed trends in admission for nonemergency admissions with a difference-in-difference approach, using four separate comparison groups: (1) matched on previous hospital 